\title{
Análisis de la aplicabilidad de la guía GTC- 180 sobre responsabilidad social en la Sala Administrativa del Consejo Superior de la Judicatura ${ }^{* 1}$
}

\section{Analysis of the applicability of the social responsibility guide GTC- 180 in the Administrative Chamber of the Supreme Judicial Council}

Recibido: 21 de junio de 2010

Revisado: 22 de agosto de 2010 Aceptado: 30 de septiembre de 2010

Luis Miguel Romero Smit" ${ }^{*}$

Juan Carlos Yepes Alzate ${ }^{* * 3}$

Universidad Santo Tomás e Icontec, Bogotá, Colombia

\section{RESUMEN}

Este artículo muestra los resultados de la investigación realizada en la Sala Administrativa del Consejo Superior de la Judicatura para identificar la aplicabilidad en esta entidad de los aspectos de la responsabilidad social propuestos por la guía colombiana GTC-180. Se utilizó inicialmente como metodología para recopilar información, la elaboración de varios tipos de encuestas que se aplicaron a los empleados de los diferentes niveles jerárquicos de la Sala, y que se elaboraron tomando como referente la GTC-180. Del análisis de estos datos se llegóa unas conclusiones preliminares que fueron debatidas con tres expertos en responsabilidad social del país, para extraer las conclusiones definitivas. Los resultados mostraron que la responsabilidad social es un elemento indispensable para la gestión de la Sala Administrativa del Consejo Superior de la Judicatura, particularmente en los aspectos relacionados con el respeto a los derechos de los

* Artículo de investigación científica resultado del proyecto de investigación.

** Correspondencia: Luis Miguel Romero Smit. Profesional en Finanzas y Relaciones Internacionales. Magíster en Calidad y Gestión Integral. CONSEJO SUPERIOR DE LA JUDICATURA. Correo electrónico: luismiguelromero@hotmail.com.

*** Juan Carlos Yepes Alzate. Abogado. Magíster en Calidad y Gestión Integral. Consejo Superior de la Judicatura. Correo electrónico: juanyepes@ hotmail.com 
empleados, los aspectos éticos (incluyendo los principios y valores de gestión, el desarrollo del código de ética y las prácticas contra la corrupción) y el gobierno corporativo.

Palabras clave: GTC - 180, responsabilidad social, rama judicial, partes interesadas.

\section{ABSTRACT}

This article shows the results of a research carried out in the Administrative Chamber of the Supreme Judicial Council to identify the applicability of the social responsibility aspects given by Colombian GTC-180. The processing of various types of surveys that were given to the officials of different hierarchical levels of the Chamber, which were developed by reference to the GTC-180, was initially used as a methodology for gathering information. The analyses of this data led to preliminary conclusions that were discussed with three experts in social responsibility in the country, to extract definitive conclusions. The results showed that social responsibility is indispensable for the management of the Administrative Chamber of the Supreme Judicial Council, particularly in matters related to the respect of civil rights, ethical issues (including the principles and management value, the development of the code of ethics and practices against corruption) and corporate goverment.

Key words: Judicial Branch, GTC-180, Social Responsibility, stakeholders.

\section{INTRODUCCIÓN}

La Rama Judicial del Poder Público representa una de las instituciones más importantes del Estado colom- biano, la cual debe caracterizarse por la transparencia en todas sus actuaciones y por un alto grado de confiabilidad en sus procesos y decisiones. Dentro de este marco, la Sala Administrativa del Consejo Superior de la Judicatura es el organismo técnico de la Rama Judicial encargado de dotarla de autonomía políticoadministrativa, y de garantizar la independencia y la autonomía funcional de quienes imparten justicia.

La Sala Administrativa desarrolla su labor por medio de programas que buscan mejorar el acceso a la justicia, ampliar la cobertura en todo el territorio nacional, adoptar medidas que eliminen la descongestión de los despachos judiciales, lograr la modernización tecnológica, incrementar la capacitación de los servidores judiciales, mejorar la eficiencia del proceso judicial mediante la implementación del sistema de oralidad y crear nuevos cargos.

Por otra parte (y pese a lo anterior, o tal vez derivado de la falta de frutos en el desarrollo de los programas arriba descritos), la Administración de Justicia en Colombia es una de las instituciones del Estado que más atención ha recibido por parte de sus asociados y la cual genera profundas preocupaciones como consecuencia de problemas estructurales, funcionales y organizacionales. De hecho, a pesar de las múltiples y profundas reformas de las que ha sido objeto, persiste en la sociedad un cierto grado de opinión que refleja insatisfacción sobre su funcionamiento y que afecta negativamente la confianza del pueblo colombiano en ella.

En este sentido, la Administración de Justicia debe caracterizarse por la transparencia, autonomía e independencia en todas sus actuaciones, un alto grado de confiabilidad en sus procesos y decisiones, y la capacidad para que todos puedan acceder en igualdad de condiciones. 
Bajo estos criterios, la Responsabilidad Social se configura como una herramienta que permite obtener resultados confiables a través del compromiso ético de la dirección y del uso de mecanismos que permiten encauzar la gestión de la organización hacia el logro de sus objetivos naturales. Por su parte, la responsabilidad social orativa actúa como una herramienta que permite obtener resultados confiables, a través del compromiso ético de la dirección y el uso de mecanismos que permiten encauzar la gestión de la organización hacia el logro de sus objetivos naturales. La responsabilidad social corporativa busca entonces alinear los objetivos y prácticas de gestión organizacionales, con las necesidades y expectativas de todas las partes interesadas con las que interactúa.

A partir de los anteriores referentes, la investigación busca determinar el alcance y las características de la responsabilidad social dentro del ámbito de la Sala Administrativa del Consejo Superior de la Judicatura, parte integral de la Rama Judicial del Poder Público en Colombia, con el fin de establecer los mecanismos de gestión adecuados en el caso de que su implementación sea factible.

Para lograr este objetivo es necesario identificar las partes interesadas en la gestión de la Sala Administrativa del Consejo Superior de la Judicatura, que pueden verse perjudicadas por fallas en la gestión o la no ejecución de sus actividades, así como determinar las categorías y variables en las que la Sala Administrativa del Consejo Superior de la Judicatura puede cumplir con características de responsabilidad social (RS) como resultado de la ejecución de sus actividades, y establecer los mecanismos de gestión para tal fin.

El logro de estos objetivos permitirá establecer cuáles son los criterios para determinar la responsabilidad social corporativa en la Rama Judicial del Poder Público en Colombia y cuáles las políticas de RS aplicables a la Rama Judicial en Colombia.

\section{METODOLOGÍA}

El enfoque metodológico corresponde a la perspectiva distributiva y a la perspectiva estructural. La primera tiene como herramienta la encuesta estadística y permite la investigación de hechos; la segunda enmarca la investigación de opiniones, ya sea mediante grupos de discusión o entrevistas, a través de la discusión de significados. Estas perspectivas se desarrollan de acuerdo con la metodología descrita por Ibáñez. Para el efecto se emplearon las siguientes herramientas: análisis de textos teóricos para la construcción del marco teóricoreferencial, encuestas a grupos focales, análisis estadístico y procesamiento de datos, entrevistas a expertos en RS, y aplicación lógica deductiva.

Las categorías de RS aplicables a la entidad objeto del estudio surgen de un marco teórico referencial construido teniendo en cuenta las características estructurales de la Sala Administrativa del Consejo Superior de la Judicatura y mediante la lectura y análisis de diversos textos que sustentan el marco teórico de la RS, de la gestión y de la administración de justicia. Hecho esto, se seleccionó la guía colombiana GTC-180 sobre responsabilidad social como referente para establecer las categorías y variables de la $\mathrm{RS}$ y se procedió al diseño de las encuestas dirigidas a empleados y empleados de la Rama Judicial, con el fin de observar la percepción que tienen unos y otros sobre la aplicabilidad y existencia de los diversos elementos de la responsabilidad social.

1 IBÁÑEZ, Jesús. Perspectivas de la investigación social: el diseño en las tres perspectivas. En: GARCÍA FERRANDO, Manuel; IBÁNEZ ALONSO, Jesús y ALVIRA MARTÍ́N, Francisco (Comps.). El análisis de la realidad social. Métodos y Técnicas de Investigación. Madrid : Alianza, 1990, p. 49 -83. 
Descripción de la organización

La estructura organizacional de la Rama Judicial del

Poder Público de Colombia se puede observar en la fi- gura 1. Dentro de esa estructura, la Sala Administrativa tiene, entre otras, las siguientes funciones:

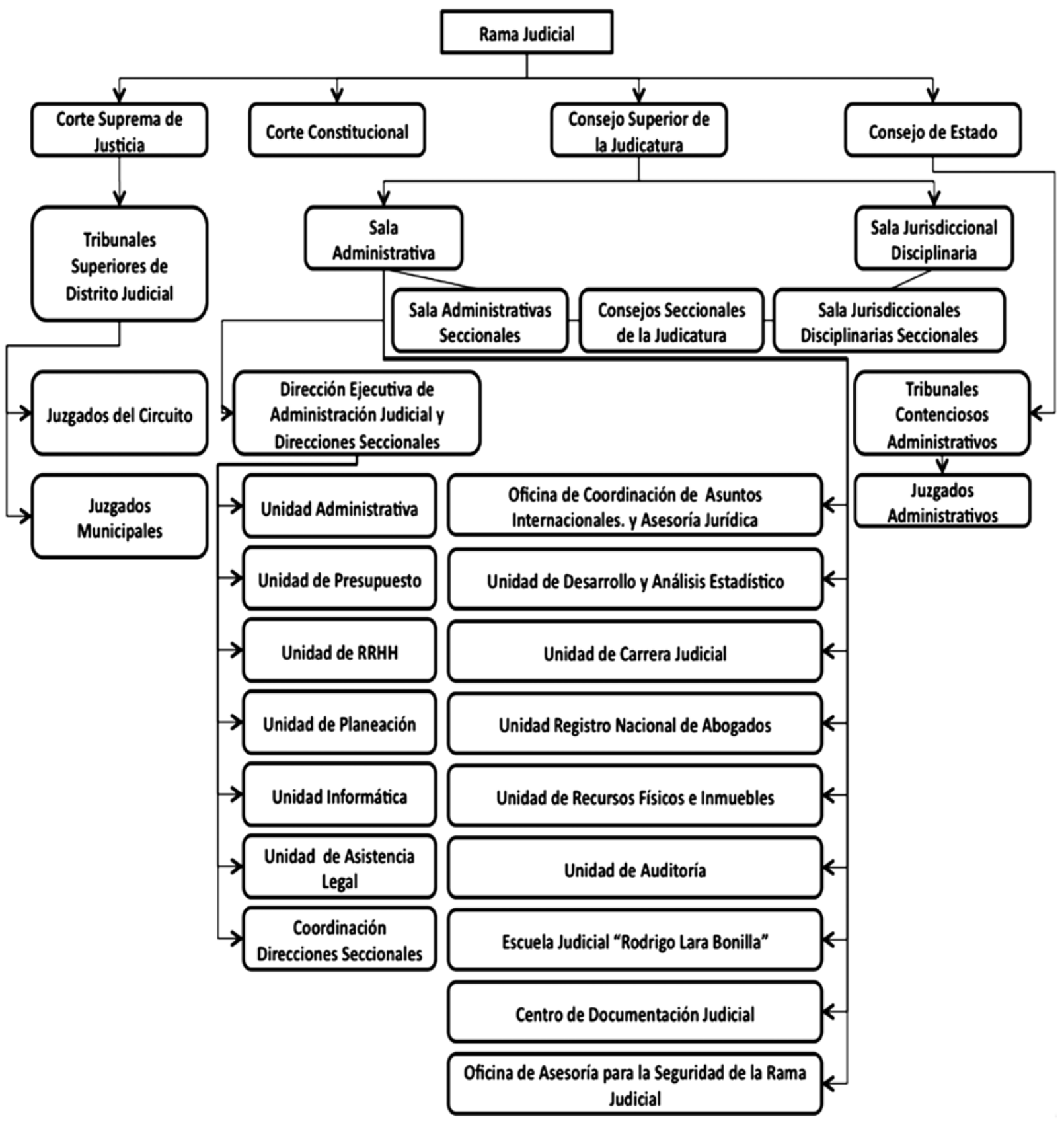

Fiǵura 1: Estructura organizacional de la Rama Judicial

Fuente: Elaboración propia. 
- Elaborar el proyecto de presupuesto de la Rama Judicial que deberá remitirse al Gobierno Nacional, el cual lo incorpora al proyecto que proponga la Fiscalía General de la Nación;

- Elaborar el proyecto de Plan Sectorial de Desarrollo para la Rama Judicial, con su correspondiente Plan de Inversiones y someterlo a la aprobación del Consejo en Pleno;

- Autorizar la celebración de contratos y convenios de cooperación e intercambio que deban celebrarse conforme a la Constitución y las leyes para asegurar el funcionamiento de sus programas y el cumplimiento de sus fines, cuya competencia corresponda a la Sala;

- Aprobar los proyectos de inversión de la Rama Judicial;

- Crear, ubicar, redistribuir, fusionar, trasladar, transformar y suprimir tribunales, las salas de éstos y los juzgados, cuando así se requiera para la más rápida y eficaz administración de justicia, así como crear salas desconcentradas en ciudades diferentes de las sedes de los distritos judiciales, de acuerdo con las necesidades de éstos;

- Fijar la división del territorio para efectos judiciales, tomando en consideración para ello el mejor servicio público;

- Determinar la estructura y la planta de personal del Consejo Superior de la Judicatura;

- Determinar la estructura y las plantas de personal de las corporaciones y juzgados. Para tal efecto podrá crear, suprimir, fusionar y trasladar cargos en la Rama Judicial, determinar sus funciones y señalar los requisitos para su desempeño que no hayan sido fijados por la ley;
- Enviar a la Corte Suprema de Justicia y al Consejo de Estado listas superiores a cinco candidatos para proveer las vacantes de magistrados que se presenten en estas corporaciones;

- Elaborar y presentar a la Corte Suprema de Justicia y al Consejo de Estado listas para la designación de magistrados de los respectivos tribunales, de conformidad con las normas sobre carrera judicial;

- Dictar los reglamentos relacionados con la organización y funciones internas asignadas a los distintos cargos;

- Regular los trámites judiciales y administrativos que se adelanten en los despachos judiciales, en los aspectos no previstos por el legislador;

- Dictar los reglamentos sobre seguridad y bienestar social de los empleados y empleados de la Rama Judicial, de acuerdo con las leyes que en la materia expida el Congreso de la República;

- Administrar la carrera judicial de acuerdo con las normas constitucionales;

- Realizar la calificación integral de servicios de los magistrados del Tribunal;

- Establecer indicadores de gestión de los despachos judiciales e índices de rendimiento, lo mismo que indicadores de desempeño para los empleados y empleados judiciales con fundamento, en los cuales se realice su control y evaluación correspondientes;

- Regular, organizar y llevar el Registro Nacional de Abogados y expedir la correspondiente tarjeta profesional;

- Establecer el régimen y la remuneración de los auxiliares de la justicia; 
- Reglamentar la carrera judicial;

- Elaborar y desarrollar el plan de formación, capacitación, y adiestramiento de los empleados y empleados de la Rama Judicial;

- Coadyuvar para la protección y seguridad personal de los empleados y de la Rama Judicial;

- Llevar el control del rendimiento y gestión institucional de la Corte Constitucional, de la Corte Suprema de Justicia, del Consejo de Estado y de la Fiscalía General de la Nación;

- Elegir al Auditor del Consejo.

De la Sala Administrativa se derivan las salas administrativas seccionales en todo el país, la Dirección Ejecutiva de Administración Judicial y las Unidades Adscritas.

Como se observa, es una organización compleja tanto en tamaño, como en disposición geográfica y diversidad de actividades, por lo que la investigación se desarrolló dentro del ámbito organizacional, geográfico y funcional de la Sala Administrativa del Consejo Superior de la Judicatura en su nivel central (ubicada en diversas locaciones en la ciudad de Bogotá), en tanto que por mandato legal es la llamada a diseñar e implementar las herramientas de gestión que definen el actuar administrativo de toda la Rama Judicial del Poder Público en Colombia.

\section{Definición de las categorías de RS aplicables a la organización}

El fundamento normativo del cual se derivan las categorías, variables y partes interesadas en RS para propósitos de la investigación es la Guía Técnica Colombiana GTC-180, norma que busca proporcionar directrices para establecer, implementar, mantener y mejorar en forma continua un enfoque de responsabilidad social en la gestión.

De igual manera, la identificación de las partes interesadas y el diagnóstico de la percepción de los miembros de la Rama Judicial sobre aquellos elementos de RS que aplican para la organización, se realizó mediante la aplicación de encuestas al personal que labora dentro de la Sala Administrativa del Consejo Superior de la Judicatura. La encuesta consta de dos partes: la primera busca establecer el orden de importancia de las partes interesadas de acuerdo con la conclusión tomada del análisis de textos relacionados. La segunda parte contiene cada uno de los componentes de la RS de acuerdo con los lineamientos de la GTC-180, con el fin de determinar la percepción de los servidores judiciales sobre la pertinencia de éstos y su importancia relativa.

\section{El diseño de la encuesta}

Dadas las preguntas de investigación, se estableció como base el concepto de responsabilidad social aplicable a la Sala Administrativa del Consejo Superior de la Judicatura. Una vez establecido el concepto principal que las preguntas de la investigación buscan resolver, se determinaron las categorías relacionadas con dicho concepto, de acuerdo con el marco referencial de la GTC-180, así:

- Partes interesadas

- Principios y valores de la gestión

- Buen gobierno corporativo

- Respeto por los derechos humanos

- Cumplimiento de las normas de protección y mejoramiento del medio ambiente

- Protección de la organización y de sus partes interesadas frente a prácticas asociadas a la corrupción 
- Contribución al desarrollo económico y social

- Respeto y protección al usuario

- Respeto a los derechos de los empleados y empleados.

Para cada una de las categorías descritas se determinaron las variables sobre las que se pregunta en la encuesta, luego se elaboraron las preguntas y se incorporaron en la encuesta a los empleados de la Sala Administrativa del Consejo Superior de la Judicatura.

La determinación del tamaño de la muestra se hizo teniendo en cuenta la planta de personal existente en el nivel central de la Sala Administrativa del Consejo Superior de la Judicatura, incluyendo la Presidencia de la Sala, la Dirección Ejecutiva de Administración Judicial y las unidades adscritas. El universo poblacional fue de 421 personas, y la muestra de 78 servidores judiciales.

El resultado de la encuesta arrojó conclusiones preliminares que fueron objeto de discusión en la entrevista a dos expertos en el tema de RS en Colombia ${ }^{2}$ y de las cuales se establecieron las conclusiones definitivas.

2 Experto consultado: Gustavo Yepes López: Administrador de Empresas de la Pontificia Universidad Javeriana, Máster en Administración de Negocios de la Universidad Externado de Colombia y aspirante al título de Doctorado en Administración de Negocios en la Universidad Antonio de Nebrija (España). Es co-autor de los libros "La responsabilidad social empresarial, fundamentos y aplicación en las organizaciones de hoy" y "Liderazgo, evolución y conceptualización", entre otros artículos e investigaciones. Actualmente es parte del Comité Técnico 180 de ICONTEC, profesor de la iniciativa intercolegiada en RS -ERSE- y miembro de la Junta Editorial de la revista RS.

Experto consultado. Ramiro Restrepo González: Presidente del Comité Técnico 180 de ICONTEC, que formuló la Guía técnica colombiana en responsabilidad social. Miembro del Comité Espejo Colombiano ante la ISO en la discusión y construcción de la futura ISO-26000 en responsabilidad social. Ex presidente de la Asociación Colombiana de Relaciones de Trabajo -Ascort-. Ha sido Gerente de Gestión Humana del Grupo Corona, Vicepresidente de Gestión Humana de Cadenalco S. A. y Director Administrativo de Cadena de Tiendas Venezolanas Cativen S. A. Actualmente se desempeña como Consultor Independiente.

\section{Definición del fundamento teórico}

Una vez establecido el marco referencial fue necesario comenzar el fundamento teórico. Para ello se tomó como base la sinopsis de teorías de RS elaborada por Garriga y Mele ${ }^{3}$.

Dentro del conjunto de teorías arriba descrito, se escoge el marco de las teorías éticas, dado que muestra mayor correlación con el propósito del Estado y sus instituciones.

\section{RESULTADOS}

Durante el desarrollo de la investigación, el análisis de textos relativos a la $\mathrm{RS}$ permitió que se comprendieran los principios, modelos y prácticas en un grado suficiente para elaborar las encuestas en los diferentes niveles de la Rama Judicial del Poder Público en Colombia. A su vez, el análisis de dichas encuestas permitió la identificación de aspectos clave para consultar a los expertos seleccionados.

Los componentes de la RS para la Rama Judicial del Poder Público en Colombia son muy similares, por no decir idénticos, a los aplicables en el sector privado; sin embargo, se encontraron diferencias de énfasis y relevancia que se pueden entender a partir de las particularidades de la organización.

3 GARRIGA, Elisabet y MELE, Domènec. Teorías de Responsabilidad Social Corporativa: Trazando el Territorio. Traducción de María Margarita Reyes financiada por la cuarta promoción del grupo 1 de Maestría en Administración de Empresas. Universidad del Valle: Cali, 2006. En: GUTIÉRREZ PRADA, Patricia. Trabajar con responsabilidad social en la gestión pública, implica tomar decisiones. Conferencia Ascolfa 2009. Encuentro Internacional de Investigación en Administración, Responsabilidad social de la administración en el mundo. Consultado el 10 de octubre de 2009 en <http://www.ascolfa.edu.co/local/MemoriasConferenciaAscolfa09/ DOC/p604.swf> 
Juan Carlos Yepes Alzate****Universidad Santo Tomás e Icontec, Bogotá, Colombia

Tabla 1: Sinopsis de las teorías de RS.

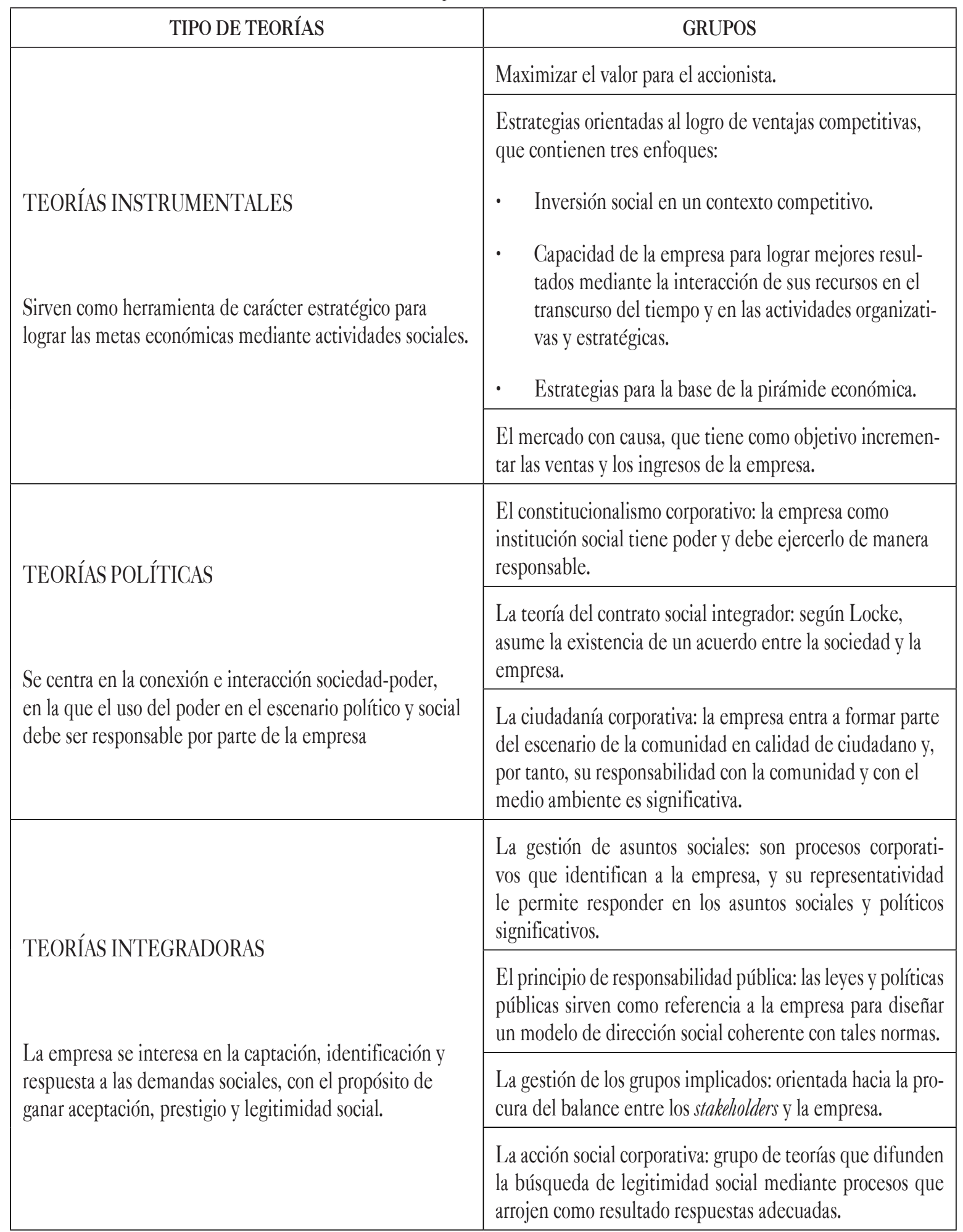


Tabla 1: Sinopsis de las teorías de RS.

\begin{tabular}{|l|l|}
\hline \multicolumn{1}{|c|}{ TIPO DE TEORÍAS } & \multicolumn{1}{c|}{ GRUPOS } \\
\hline \multirow{5}{*}{ TEORÍAS ÉTICAS } & $\begin{array}{l}\text { La teoría normativa de grupos implicados: que trata sobre } \\
\text { las obligaciones fiduciarias de la empresa con todos sus gru- } \\
\text { pos interdependientes. }\end{array}$ \\
\cline { 2 - 2 } $\begin{array}{l}\text { Se fundamentan en la responsabilidad ética de la empresa } \\
\text { con la sociedad, sus derechos y el medio ambiente, orien- } \\
\text { tada a la construcción de una sociedad mejor. }\end{array}$ & $\begin{array}{l}\text { El desarrollo sostenible: que consiste en alcanzar el desa- } \\
\text { manos y laborales y el respeto al medio ambiente. } \\
\text { vo de preservarlo para las generaciones presentes y futuras. }\end{array}$ \\
\cline { 2 - 2 } & $\begin{array}{l}\text { El enfoque del bien común: la empresa como parte de la } \\
\text { sociedad debe aportar al bien común de la sociedad en que } \\
\text { se desarrolla y, de igual manera, aportar al bienestar social } \\
\text { y a la convivencia bajo las condiciones de justicia, paz y } \\
\text { amistad en el presente y para el futuro. }\end{array}$ \\
\hline
\end{tabular}

Los componentes más relevantes apuntan al desarrollo de políticas y prácticas que deben atender las expectativas y necesidades principalmente hacia los empleados (nivel interno) y en segundo lugar hacia los usuarios (nivel externo), seguidos del respeto por los derechos humanos, la protección a los usuarios y la gestión ética, la trasparencia y la lucha contra la corrupción. A diferencia de lo que se puede presentar en una organización privada, la protección medioambiental y el aporte al desarrollo económico son componentes con menor relevancia.

La opinión de los expertos consultados coincide con los datos obtenidos en las encuestas realizadas a empleados de la Rama Judicial en relación con la factibilidad de aplicar en forma prioritaria los principios, modelos y técnicas de la RS con los empleados y los usuarios; sin embargo, no se presenta el mismo acuerdo con lo relacionado en el manejo medioambiental.

La selección de los principios y valores organizacionales identificados en las encuestas son manifestación de situaciones por resolver en la relación entre la alta dirección y los empleados, es decir, que manifiestan conflictos subyacentes de respeto y equidad en el trato a través de los niveles de la organización, según se desprende de las respuestas dadas a las preguntas relacionadas con el escalafón de los principios y valores que serían los más importantes para la organización.

De acuerdo con los resultados de las encuestas, las partes interesadas más importantes son los empleados y empleados de la organización, seguido por los usuarios del servicio de justicia y los proveedores de servicios tecnológicos, mientras que el gobierno y la alta dirección juegan un rol secundario, y las comunidades son un elemento de poca relevancia.

Los expertos consultados coinciden en la apreciación relacionada con el papel de los empleados, sin embargo, hay discrepancias en el rol de las comunidades. Ramiro Restrepo le da un rol fundamental al ciudadano, homologándolo al papel del consumidor en el sector privado. Igualmente, le da mayor valor al papel del Congreso de la República al señalar el entorno completamente regulado en el que se mueve la 
organización, lo cual afecta la definición misma de RS para el sector público, pues ésta implica ir más allá de lo exigido por las normas.

La alta dirección y las organizaciones de control tienen un rol secundario frente a los empleados y los usuarios del servicio de justicia, y los menos importantes del ranking son las comunidades, tanto del nivel central como seccional, seguidos de las familias y organizaciones de los empleados.

Se evidencia una debilidad global en el estilo de dirección, en la medida en que ninguna de las variables evaluadas tuvo una aceptación frente a la realidad de la organización, superior al 80\%.

El resultado indica que las mayores falencias frente a las variables de gobierno corporativo de la organización, en opinión de los encuestados, se relacionan con que los directivos no tienen en cuenta los conocimientos y habilidades de sus colaboradores para asignar las cargas de trabajo, el estilo de dirección no facilita la participación de los servidores públicos, no existe compromiso de la alta dirección con el diseño y cumplimiento de políticas y prácticas de desarrollo del talento humano y el estilo de dirección no facilita la resolución de conflictos internos y externos. También se menciona que los directivos no tienen los conocimientos y destrezas para dirigir las áreas bajo su responsabilidad y la alta dirección no facilita la comunicación y retroalimentación entre los niveles de la organización.

Si bien hay una apreciación de que el respeto por los derechos humanos es parte integral de la misión, las políticas y la planeación estratégica de la Rama Judicial, no se considera que ésta fomente en su personal, en forma abierta e inequívoca el respeto por los derechos humanos de los usuarios del servicio de justicia (cerca de un 39\% está en desacuerdo).
En tal sentido, la organización ha efectuado un análisis de las interacciones con sus usuarios en las diferentes etapas de realización de sus productos y servicios, sin embargo, su difusión ha sido parcial en la medida en que un $31 \%$ de los encuestados todavía no conoce la existencia de dicho análisis.

Igualmente, cerca del $80 \%$ de los encuestados no sabe o no responde o se considera en desacuerdo con la afirmación "la Rama Judicial se preocupa por proporcionarle al usuario información sobre aspectos de costos e implicaciones ambientales de sus productos y servicios", lo cual refuerza la necesidad de iniciar la revisión de los aspectos ambientales significativos de la organización, para así desarrollar e implementar las herramientas más adecuadas de mitigación de impactos ambientales.

En el orden de importancia de elementos de buen trato a los empleados, de acuerdo con los resultados de la encuesta, prima la estabilidad laboral, la formación y el desarrollo de habilidades y competencias, el bienestar social y la salud ocupacional.

El mejoramiento de las condiciones salariales es un factor de gran importancia en la opinión de los encuestados, pero hay otros elementos que tienen mayor relevancia de acuerdo con los resultados de la encuesta, como son la formación de habilidades y competencias y la estabilidad laboral.

Se evidencia un gran desconocimiento de la existencia de un código de ética que contiene los acuerdos, compromisos o protocolos éticos, con un $76 \%$ de respuesta negativa, pese a que dicho código fue promulgado a comienzos del 2009.

Si bien se observa que hay un acuerdo en que el código fue elaborado mediante el diálogo y participación de empleados y directivos, y que se han puesto en marcha políticas y estrategias de gestión para aplicarlo, 
una tercera parte de los que respondieron positivamente están en desacuerdo con que para la solución de los conflictos internos se consideren los parámetros del código de ética, y que se difundan los acuerdos, compromisos o protocolos éticos a la ciudadanía o a las partes interesadas.

La mayoría de las respuestas indica que los ámbitos que demuestran el control ético son desconocidos o se considera que no son aplicados adecuadamente dentro de la organización.

De acuerdo con los resultados de la encuesta, la percepción global y resumida por orden de importancia de las variables que comprenden el desarrollo económico y social en los que la Rama Judicial podría participar 0 no prioriza el desarrollo tecnológico, el mejoramiento de las condiciones salariales, el incremento del índice de educación nacional y la retención de empleados experimentados y formados, lo que es también un indicativo de la expectativa de la organización por atender en principio las necesidades internas y darles relevancia secundaria a las expectativas externas.

Según los resultados, la percepción es que no existen criterios de medición para verificar el cumplimiento de las políticas en materia de respeto a los derechos humanos. De la misma forma, no existe acuerdo sobre la existencia de mecanismos mediante los cuales cualquier parte interesada pueda expresar su inconformidad con las actividades de la Rama Judicial respecto al manejo de los derechos humanos.

Se aprecia un desacuerdo sobre las herramientas ambientales, manifestado a través de una combinación entre las respuestas "inadecuada" y "no sabe/no responde" las cuales tienen un rango que abarca desde un 36\% a un 62\% del acumulado de las herramientas. Teniendo en cuenta lo anterior, el escalafón de la opinión sobre las herramientas ambientales más adecuadas para la Rama Judicial es el siguiente:
- Políticas de reciclaje

- Tecnologías para el ahorro de energía

- Manejo de la disposición de residuos sólidos

- Control de vertimientos (líquidos contaminantes)

- Control de emisiones atmosféricas

Hay un desconocimiento general sobre el manejo que debería darle la Rama Judicial al tema ambiental, lo que indica que la Rama Judicial debería prestar más atención a sus aspectos ambientales.

Los mecanismos de gestión específicos para que la Sala Administrativa del Consejo Superior de la Judicatura pueda cumplir con las categorías y variables de la RS no se alcanzaron a definir, debido a que no fue posible discutir este tema en entrevistas con los expertos en el manejo y la administración de la justicia en Colombia, tal como lo son los magistrados, sin embargo, en el capítulo de recomendaciones se presentan algunos lineamientos para hacerlo, los cuales surgen del análisis documental efectuado y de las entrevistas realizadas con los expertos.

\section{Categorías y variables de $\mathrm{RS}$ aplicables para la Rama Judicial}

\section{Gobierno corporativo}

Es una de las categorías más relevantes dentro de la $\mathrm{RS}$, pues representa la concreción de los principios y valores que debe manifestar la alta dirección. Se define como la filosofía y el modo de administrar del gerente público, caracterizada por competencia, integridad, transparencia y responsabilidad pública. 
En esta categoría es necesario que se refuercen variables como el manejo de los conocimientos y habilidades de los servidores judiciales a la hora de asignar las cargas de trabajo, y facilitar mecanismos para permitir la participación de los servidores públicos, la resolución de conflictos y la comunicación y retroalimentación entre los niveles de la organización.

\section{Respeto por los derechos humanos}

No se debe demeritar la acción dirigida a la comunidad, particularmente en la protección de su modo de vida socioeconómico. En este sentido, se hace necesario diseñar mecanismos de divulgación y retroalimentación de las actividades que se desarrollen, con el fin de disminuir la percepción de apatía hacia el tema, por parte de los servidores judiciales.

\section{Medio ambiente}

Tradicionalmente, el tema medioambiental no ha sido objeto de mayor atención por la alta dirección o los servidores judiciales, sin embargo, esto también puede deberse a la falta de estudios técnicos sobre los aspectos e impactos ambientales relacionados con la organización. Es particularmente notorio que la percepción de los servidores judiciales indique que las principales herramientas de manejo medioambiental sean la implementación de políticas de reciclaje y el uso de tecnologías para el ahorro de energía, en consonancia con la naturaleza de las actividades desarrolladas por la Rama Judicial.

Es necesario, entonces, realizar una revisión de los aspectos ambientales y proceder a plantear las medidas específicas según las prioridades aquí descritas. Igualmente, se hace evidente la necesidad de manifestar en forma clara el compromiso de la organización hacia prácticas amigables con el medio ambiente y revisar la legislación aplicable al respecto.

\section{Gestión ética}

Esta es la categoría más relevante en RS aplicable a la Rama Judicial, pero al mismo tiempo es la menos abordada por la organización. Cerca del $76 \%$ de los encuestados desconoce de la existencia de un código de ética que contiene los principios y protocolos éticos convenidos por la organización, pese a que fue adoptado desde el año 2008. Igualmente, se evidencian serias fallas en su diseño, pues no se utilizó la metodología aprobada para entidades del Estado, y también en su implementación, ya que no se utiliza para la resolución de conflictos internos o externos.

La percepción negativa de los propios servidores judiciales sobre el uso de herramientas para controlar la información al prevenir situaciones de corrupción o la imagen negativa de la organización en materia de lucha contra la corrupción, es motivo de preocupación. Por lo anterior, se hace imperioso divulgar los principios y prácticas aprobados dentro del código de ética, empoderar a los empleados en su uso continuo y aplicación para la toma de decisiones.

\section{Desarrollo económico y social}

Existe cierto desacuerdo entre los encuestados sobre si esta categoría debe hacer parte de un esquema de RS en la Rama Judicial; en caso de incorporarse dentro de cualquier serie de políticas y planes en RS, esta categoría deberá dar prelación a aspectos internos tales como el mejoramiento de las condiciones salariales de los servidores judiciales y la retención de empleados experimentados y formados, y dejar de lado aspectos como la inclusión de minorías, la protección social 0 el incremento de la productividad del capital humano. 


\section{Respeto y protección al usuario}

En tanto que esta categoría puede abordarse desde la perspectiva de la satisfacción de las necesidades de los usuarios y la transparencia en la gestión institucional, puede ser abordada por las entidades del Estado, ya no sólo como elemento de libre adopción, sino por imposición legal a través de las normas de implementación de sistemas de gestión de calidad para el sector público y modelos de control interno que abogan por la rendición de cuentas.

En dicho aspecto, la Rama Judicial se encuentra en el proceso de implementación de las herramientas, con éxito parcial, puesto que si bien existe un sistema de gestión certificado y en funcionamiento, buena parte de los servidores judiciales encuestados no conocen los elementos que integran este sistema, sus objetivos 0 alcances.

Por lo anterior, se hace necesario reforzar los mecanismos de divulgación y comunicación con el fin de que los servidores judiciales se empoderen dentro de la aplicación del sistema de gestión de calidad y reconozcan los beneficios inherentes a su uso.

\section{Respeto y protección al trabajador}

Esta es una de las categorías con mayor grado de importancia percibida por los servidores judiciales encuestados, los cuales enfocan su interés en la estabilidad laboral, el mejoramiento de la salud ocupacional y el desarrollo de habilidades y competencias. En segundo plano quedan el aspecto salarial y prestacional, y ofrecen poca atención a aspectos que aparentemente deberín tener más relevancia, tales como la libertad de asociación y el mejoramiento de las condiciones del núcleo familiar de los empleados.
Esta distribución puede explicarse por la tendencia hacia la profesionalización de la planta de personal, que obliga a los empleados a mejorar sus competencias con el fin de acceder a puestos en carrera administrativa, que aseguran cierto grado de estabilidad según el desempeño mostrado.

\section{CONCLUSIONES}

La implementación de esquemas de responsabilidad social en el sector público es beneficiosa en la medida que permite:

- Identificar en forma objetiva a las partes interesadas de la organización;

- Crear modelos de dirección y gestión participativas, coadyuvar o incrementar la transparencia y rendición de cuentas públicas;

- Generar el compromiso ético de los empleados y directivos;

- Incrementar el respeto hacia la diversidad;

- Promover la igualdad de oportunidades, y

- Favorecer la adopción de códigos formales de responsabilidad social que articulan el diálogo y establecen políícas y criterios de seguimiento y control.

A partir de los resultados de la investigación se deben establecer mecanismos de gestión que permitan la implementación eficaz de todos los componentes expuestos, pero dichos mecanismos no son abordados de forma directa en esta investigación. Sin embargo, se sugiere el uso de la hoja de ruta de cinco pasos, propuesta por el experto Ramiro Restrepo (ver Anexo A). 
Por lo anterior, se espera que esta investigación sirva de base para determinar las características de la RS en otras organizaciones similares del sector público, y como punto de partida para el establecimiento de políticas, criterios, prácticas específicas y mecanismos de control y seguimiento en materia de RS dentro de la Rama Judicial en Colombia como en el resto del Estado colombiano.

Al hacer una revisión de los resultados aquí expuestos es válido concluir que la responsabilidad social no sólo es útil, sino también necesaria dentro de las entidades públicas de Colombia y, particularmente, en la Rama Judicial del Poder Público, porque constituye un norte claro en la creación de políticas públicas, ayuda en la priorización de planes y programas de gestión institucional, en la medida que aporta a la construcción de la transparencia y la ética pública de los servidores judiciales que integran la Entidad y a la reconstrucción de la confianza del tejido social en sus instituciones.

Es de aclarar que en la aplicación del modelo establecido en la GTC-180 (o cualquier otro modelo de RS) y en las demás Altas Cortes de la Rama Judicial, los resultados pueden ser diferentes a los obtenidos en la presente investigación.

Para desarrollar el análisis en las demás cortes, es necesario cambiar ciertas condiciones, tales como el acceso y comunicación con la alta dirección, elaborar marcos teórico-referenciales específicos para cada alta corte dada la naturaleza divergente de sus funciones, determinar las partes interesadas específicas y comunes y las interacciones entre las mismas Cortes, y evaluar el grado en que la gestión de éstas depende de las políticas emanadas por la Sala Administrativa del Consejo Superior de la Judicatura, así como el grado de autonomía de dicha gestión.

\section{RECOMENDACIONES}

Cualquier política por desarrollar en materia de RS deberá dar prevalencia al respeto por los derechos humanos, el respeto al usuario y el respeto a los empleados.

El cumplimiento de las normas medioambientales, el gobierno corporativo y la contribución al desarrollo económico deberán ser aspectos subsidiarios dentro de esta política.

Dicha política deberá otorgar mayor relevancia a valores tales como la tolerancia, la confianza y el cumplimiento de la normatividad vigente.

De igual forma, deberá atender la necesidad de mejorar la percepción sobre la situación del gobierno corporativo de la Rama Judicial, tanto a través de los comportamientos de la alta dirección, como de los elementos éticos.

Igualmente, entre los diversos esquemas y herramientas de RS en los temas medioambientales, de derechos humanos y desarrollo económico y social, las políticas de RS deberán dar preferencia a aquellos que favorezcan directamente a los empleados.

Será materia de una investigación específica definir el grado de descontento existente entre los empleados en relación con el trato recibido por los niveles altos y medios de la entidad, con el fin de establecer con claridad si implica una creencia subyacente de que en la actualidad no hay equidad en el trato o si se puede interpretar que en realidad este valor es manifestado activamente por los empleados.

La respuesta negativa al compromiso de la alta dirección con el diseño y cumplimiento de políticas y prácticas de desarrollo del talento humano requiere determinar inicialmente cuáles son las prácticas y políticas de desarrollo del talento humano que debe 
suscribir la alta dirección, para iniciar posteriormente su implementación.

Dicha labor puede ser objeto de una investigación específica relacionada con el estado del recurso humano en cuanto a capacitación, formación y experiencia, la cual tenga en cuenta también las inquietudes, frustraciones y anhelos del personal de la organización.

En materia de solución de conflictos, es necesario estudiar los esquemas de comportamiento vigentes en la alta dirección, por medio de un análisis de la cultura organizacional.

De igual forma, la resolución de conflictos se manifiesta tanto hacia dentro (negociación con sindicatos) como hacia fuera de la organización (choques con los otros poderes del Estado).

Los requisitos de ingreso para la alta dirección no incluyen temas de manejo del talento humano como habilidades en liderazgo, trabajo en equipo o conocimientos específicos en temas administrativos. Por tanto, deben implementarse mecanismos para corregir esta situación desde el momento del análisis de las hojas de vida de los candidatos a seleccionar.

Desde este punto de vista, la implementación de sistemas de gestión de calidad en las organizaciones del Estado puede servir como herramienta para lograr dicho objetivo.

Se debe realizar una investigación adicional con el fin de determinar si se aplican las prácticas para el respeto de los derechos humanos indicadas en la investigación.

La misma situación aplica para establecer los criterios de medición utilizables para verificar el cumplimiento de las políticas en la materia, su aplicabilidad y el resultado de su cumplimiento.
La implementación de sistemas de gestión de calidad puede servir como herramienta para atender adecuadamente las necesidades y expectativas de los usuarios en materia de derechos humanos.

Se hace necesario verificar la correcta implementación del sistema para la atención en Peticiones Quejas y Reclamos (PQR) como parte de los mecanismos para que los usuarios puedan expresar su inconformidad con las actividades de la Rama Judicial respecto al manejo de los derechos humanos, y hacer un análisis de sus resultados con posterioridad.

Se debe iniciar la revisión de los aspectos ambientales significativos de la organización, para así desarrollar e implementar las herramientas más adecuadas de mitigación de impactos ambientales.

Es materia de una investigación específica el determinar las causas de que el mejoramiento de las condiciones laborales de los empleados ocupe el último lugar en la percepción de importancia de los encuestados, lo cual se manifiesta en el hecho que no existe una asociación sindical activa dentro de la Sala Administrativa del Consejo Superior de la Judicatura.

Es necesario profundizar mediante otra investigación específica las razones de la ubicación de la percepción de la importancia de la actividad sindical en el seno de la Sala Administrativa del Consejo Superior de la Judicatura, con el fin de determinar qué origina dicha situación y si amerita tomar correctivos al respecto.

Las políticas de RS en la Rama Judicial deberán fomentar y mejorar el desarrollo en la implementación de sistemas de Gestión de Calidad, para garantizar la búsqueda de la satisfacción de los usuarios, en la medida en que no hay una socialización efectiva de los resultados ni de aspectos detallados de su funcionamiento y objetivos. 


\section{BIBLIOGRAFÍA}

IBÁÑEZ, Jesús. Perspectivas de la investigación social: el diseño en las tres perspectivas. En: GARCÍA FERRANDO, Manuel; IBÁNEZ ALONSO, Jesús y ALVIRA MARTÍN, Francisco (Comps.). El análisis de la realidad social. Métodos y técnicas de investigación. Madrid: Alianza, 1990, p. 49 -83.

GARRIGA, Elisabet y MELE, Domènec. Teorías de responsabilidad social corporativa: Trazando el territorio. Traducción de María Margarita Reyes financiada por la cuarta promoción del grupo 1 de Maestría en Administración de Empresas. Universidad del Valle: Cali, 2006. En: GUTIERREZ PRADA, Patricia. Trabajar con responsa- bilidad social en la gestión pública, implica tomar decisiones. Conferencia Ascolfa 2009. Encuentro Internacional de Investigación en Administración, Responsabilidad social de la administración en el mundo. Consultado el 10 de octubre de 2009 en http://www.ascolfa.edu.co/local/MemoriasConferenciaAscolfa09/DOC/p604.swf

\section{ANEXOS}

Anexo A. Mapa para la implementación de la responsabilidad social

Este esquema fue aportado por el profesor Ramiro Restrepo, y se publica con su autorización.

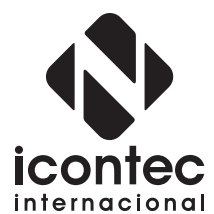

\section{MAPA DE IMPLEMENTACIÓN DE UN ENFOQUE RS}

\begin{tabular}{|c|c|c|c|c|}
\hline ACCIONES & ACCIONES & ACCIONES & ACCIONES & ACCIONES \\
\hline $\begin{array}{l}\text { - Líder } \\
\text { - Comité de RS } \\
\text { - Política de RS } \\
\text { - Modelo de RS } \\
\text { - Haga público } \\
\text { - Plan de Trabajo } \\
\text { - Planes de apoyo } \\
\text { - Antecedentes } \\
\text { - Autodiagnóstico } \\
\text { - Diagnóstico interno } \\
\text { - Diagnóstico externo } \\
\text { - Alineación de } \\
\text { proyectos }\end{array}$ & $\begin{array}{l}\text { - Asegure ganancias } \\
\text { tempranas: ACPM } \\
\text { - Defina proyectos } \\
\text { tácticos de RS } \\
\text { - Defina el proyecto } \\
\text { estratégico de RS } \\
\text { - Alinéese con el } \\
\text { sector social. } \\
\text { Haga alianzas }\end{array}$ & $\begin{array}{l}\text { - Identifique, } \\
\text { caracterice y priorise } \\
\text { sus partes } \\
\text { - Defina estrategias } \\
\text { - Diseñe mecanismos } \\
\text { de interlocución y } \\
\text { concertación } \\
\text { - Diseñe } \\
\text { procedimientos } \\
\text { de interlocución } \\
\text { y concertación }\end{array}$ & $\begin{array}{l}\text { - Adopte un } \\
\text { estándar } \\
\text { - Alinee su métrica y } \\
\text { sus sistemas de } \\
\text { información } \\
\text { - Involucre a sus } \\
\text { partes interesadas } \\
\text { - Publique una } \\
\text { memoria de } \\
\text { sostenibilidad } \\
\text { - Someta su memoria } \\
\text { a concepto de tercera } \\
\text { parte }\end{array}$ & $\begin{array}{l}\text { - Adopte un modelo } \\
\text { - Conduzca } \\
\text { autoridades internas } \\
\text { y externas de RS } \\
\text { - Divulgue los } \\
\text { resultados } \\
\text { - Reaccione los } \\
\text { resultados } \\
\text { - Reaccione ante los } \\
\text { resultados y de a } \\
\text { conocer sus planes } \\
\text { de mejora a sus } \\
\text { partes interesadas }\end{array}$ \\
\hline
\end{tabular}

